\title{
Evolution of cancer DNA methylotyping
}

\author{
Vladimir V Strelnikov*,1,2 (iD) \& Dmitry V Zaletaev1,2,3 \\ ${ }^{1}$ Epigenetics Laboratory, Research Centre for Medical Genetics, Moscow, Russia \\ ${ }^{2}$ Molecular \& Cell Genetics Department, Pirogov Russian National Research Medical University, Moscow, Russia \\ ${ }^{3}$ Medical Genetics Laboratory, IM Sechenov First Moscow State Medical University (Sechenov University), Moscow, Russia \\ *Author for correspondence: vstrel@list.ru
}

First draft submitted: 26 March 2019; Accepted for publication: 9 April 2019; Published online: 30 May 2019

Keywords: 5-methyldeoxycytidine $\bullet$ cancer $\bullet$ DNA methylation • epigenomics $\bullet$ reduced representation bisulfite sequencing

In 1942, a British developmental biologist, paleontologist, geneticist, embryologist and philosopher Conrad Hal Waddington opened the avenue of modern epigenetic and epigenomic research by simply combining two terms, epigenesis and genetics. "Epigenetics is the branch of biology which studies the causal interaction between genes and their products, which bring the phenotype into being" [1]. No one could have imagined at that time how exciting and fruitful this new science would be. Although the definition applies best to embryo development and early ontogenetic stages, the scientific thought could not restrict itself to embryology only. Modern epigenomics resembles a multistory skyscraper which we have not yet fully explored but what we are already aware of is that it is sophisticatedly equipped with all means (elevators, stairs, etc.) to communicate the levels with each other; the levels known to us now being DNA methylation, including that not only at $\mathrm{CpG}$, but also at $\mathrm{CpH}$ and $\mathrm{ApH}$; cytosine modifications, such as $5 \mathrm{hmC}, 5 \mathrm{fC}$ and $5 \mathrm{caC}$; the histone code; DNA methylation sensitive and DNA methylation insensitive transcription factors and protein and ribonucleoprotein modifying complexes; mRNA and ncRNA editing; transcription-induced splicing; RNA interference; and a huge number of noncoding RNAs, which connect and regulate all epigenetic components. All of the above factors are involved in regulating ontogeny and the response to 'external and internal stimuli', while research is in progress, and it is difficult to envision what new epigenetic factors will be discovered and what roles they will play.

Still DNA methylation remains the first known and one of the main factors of epigenetic gene expression regulation, and its diagnostic and prognostic potentials are far from completely understood. In 1948, a distinguished American biochemist and geneticist Rollin Hotchkiss isolated 5-methylcytosine in higher eukaryotic DNA (calf thymus DNA) [2], although the fifth nucleotide 5-methyldeoxycytidine was first described in tubercle bacillus DNA almost a hundred years back from now, in 1920s by American chemists Treat Baldwin Johnson and Robert De Wolf Coghill (later an outstanding leader in the development of penicillin production) [3]. Russian molecular biologist Boris Fedorovich Vanyushin determined the nature of DNA sequences subject to methylation in various species and assumed that DNA methylation regulates genetic processes in the cell as early as 1959, although the first publication in Nature appeared 10 years later [4]. British molecular biologist Robin Holliday explained the role that DNA methylation plays in regulating the gene function, proposed the term 'epimutation' [5], and, in 1979, suggested the contribution of DNA methylation to carcinogenesis [6].

If there is a biological phenomenon, such as DNA methylation, there should be methods to investigate it. Once methylation sensitive restriction endonucleases have been discovered, cleavage with the HpaII and MspI isoschizomeric enzymes provides the first step in analyzing the methylation pattern of any DNA [7]. The analytical potential of PCR (a HpaII-PCR assay) for DNA methylation analysis was first mentioned in 1990 [8]. In our laboratory, we have also introduced methylation sensitive restriction enzyme PCR (MSRE-PCR) protocols in 1990s, and applied them to diagnose abnormal DNA methylation in genetic disease and to characterize the methylotypes of various cancers $[9,10]$.

At the same time, apart from MSRE-PCR, bisulfite PCR became widely used in molecular genetics [11] and, as far as it became apparent by early 2000s that abnormal DNA methylation is a marker of virtually any cancer, temptation emerged to use these simple methods in cancer molecular diagnostics. Yet, single-gene methylation

Future $\because$ Medicine 
assays were highly informative for a very limited set of tumor types, such as GSTP1 methylation informative in $80-90 \%$ of prostate cancer cases or $M G M T$ epigenetic silencing in gliomas conferring enhanced sensitivity to alkylating drugs [11]. A very successful experience was gained by a now well-known Catalonian epigeneticist Manel Esteller with colleagues, who hypothesized that, combining three to four DNA methylation markers, one can dramatically increase sensitivity of a cancer diagnostic panel. Indeed, their four-pan-cancer-gene methylation panels provided sensitivity from 68 to $88 \%$ in identifying cancers as such including breast, colorectal, lung, renal and others [11]. Yet, these panels would fail in distinguishing particular cancer types as they were assembled from a limited set of markers representing 12 genes, $p 16^{I N K 4 a}, p 15^{I N K 4 b}, p 14^{A R F}, p 73, A P C, B R C A 1$, hMLH1, GSTP1, $M G M T, C D H 1, T I M P 3$ and $D A P K$, each known to suffer abnormal epigenetic silencing in virtually any cancer.

As far as pan-cancer abnormally methylated genes were unsuitable as markers that would potentially discriminate cancer types, and knowledge of cancer biology was still insufficient to confidently pick candidate regions, a cohort of methods naturally emerged to provide unbiased search for differentially methylated loci in cancer genomes [12]. Application of such methods produced results rather surprising for the time. First, new functional classes of genes were discovered that were not expected to be specifically methylated in cancers based on the previous concepts, such as genes encoding ion channels, loosely characterized transmembrane proteins, nerve differentiation and growth regulators, as well as the genes of completely unknown functions and even deep intergenic regions containing functionally uncharacterized genomic elements $[12,13]$. Second, it became apparent that the exact positions of the regions abnormally methylated in cancer were not necessarily coincident with the promoters, readily positioning not only in $5^{\prime}$ UTRs and around the TSSs, but in the gene bodies and $3^{\prime}$ UTRs as well, and, again, in the intergenic regions thousands of base pairs from the nearest genes $[12,14]$. Despite the scientific importance of the findings achieved, extensive employment of unbiased screening methods was hampered by the troublesome physical mapping of the identified differentially methylated DNA fragments.

Demand for the unbiased genome-scale approach in differential DNA methylation analysis has become one of the key challenges proposed in the very first issues of the Epigenomics journal a decade ago [12,15]. It was the technological revolution that made it possible to widely screen multiple samples by providing the opportunity of genome-wide DNA methylation analysis with simultaneous mapping of the differentially methylated regions.

The technological revolution allowing high-throughput omics analysis has promoted not only methylomics, but also the studies of virtually all levels of molecular organization of life, including all those comprising the known apparatus of epigenomic regulation. Multiparametric integrative analyses of chromatin immunoprecipitationsequencing (ChIP-seq) on multiple histone modifications, RNA-sequencing (RNA-seq) and DNA methylation now define epigenomic landscapes for subtypes of cancers, which can predict their relative aggressiveness and survival, and highlight potential targets for future treatment approaches $[16,17]$. It has been shown that the methylome profiles correlate well with such comprehensive molecular portraits [14,16-18] even at the level of knowledge achievable with a rather selective method of microarray-based methylome analysis. The authors of the studies involving epigenomic subtyping of the tumors emphasize that their results not only suggest that DNA methylation plays critical roles in tumorigenesis but may potentially be proposed as a diagnostic biomarker for prognostication and clinical stratification of patients, and making therapeutic decisions $[14,18,19]$. We suggest that a more purposeful approach such as genome-wide bisulfite sequencing of the $\mathrm{CpG}$ islands, the regions thought to be most important for the regulation of genes expression by DNA methylation, is destined to provide even more copious and reliable methylome profiling with high biological relevance [20]. In this issue of Epigenomics, we present the first experience of obtaining reduced representation bisulfite sequencing (RRBS) data on a significant collection of clinical samples by an XmaI-RRBS approach that was developed with emphasis on effective reduction of the fraction of the genome to be sequenced without significant loss of the CpG islands. The results demonstrate for the first time good correlation between the methylotypes identified by unsupervised clustering of the RRBS data with the intrinsic subtypes of the breast cancer samples under study, suggesting that genome-wide bisulfite sequencing of the CpG islands is the method of choice in the characterization of cancer methylotypes with the perspective of diagnostic markers development.

In conclusion, a consequence of bright discoveries achieved by scientists from all around the world within almost a hundred years granted us knowledge of the 'fifth base' as such, and of its role in the epigenetic regulation in norm and disease including cancer. On the other hand, a long and somewhat uneasy evolution of the methods for DNA methylation assessment has finally produced genome-wide assays applicable for unbiased differential methylation screening in significant collections of clinical samples. Integration of genomic, transcriptomic and epigenomic data now effectively reveals cancer subtypes, and this knowledge has a strong potential of being translated into the 
clinical setting. DNA methylomes demonstrate correlation with these subtypes and, being easily detectable in the laboratories, present the perfect substrate for the development of diagnostic assays that would attribute a certain tumor sample to a well-characterized cancer subtype.

\section{Financial \& competing interests disclosure}

The research was carried out within the state assignment of Ministry of Science and Higher Education of the Russian Federation. The authors have no other relevant affiliations or financial involvement with any organization or entity with a financial interest in or financial conflict with the subject matter or materials discussed in the manuscript apart from those disclosed.

No writing assistance was utilized in the production of this manuscript.

\section{References}

1. Waddington CH. The Epigenotype. Endeavour 18-20 (1942); Reprinted in: Int. J. Epidemiol. 41(1), 10-13 (2011).

2. Hotchkiss RD. The quantitative separation of purines, pyrimidines, and nucleosides by paper chromatography. J. Biol. Chem. 175(1), 315-332 (1948).

3. Johnson TB, Coghill RD. Researches on pyrimidines. C111. The discovery of 5-methyl-cytosine in tuberculinic acid, the nucleic acid of the tubercle bacillus. J. Am. Chem. Soc. 47(11), 2838-2844 (1925).

4. Vanyushin BF, Belozersky AN, Kokurina NA, Kadirova DX. 5-Methylcytosine and 6-methylamino-purine in bacterial DNA. Nature 218(5146), 1066-1067 (1968).

5. Holliday R, Pugh JE. DNA modification mechanisms and gene activity during development. Science 187(4173), $226-232$ (1975).

6. Holliday R. A new theory of carcinogenesis. Br. J. Cancer 40(4), 513-522 (1979).

7. McClelland M, Nelson M. The effect of site-specific methylation on restriction endonucleases and DNA modification methyltransferases-a review. Gene 74(1), 291-304 (1988).

8. Singer-Sam J, LeBon JM, Tanguay RL, Riggs AD. A quantitative HpaII-PCR assay to measure methylation of DNA from a small number of cells. Nucleic Acids Res. 18(3), 687 (1990).

9. Strel'nikov VV, Nemtsova MV, Chesnokova GG et al. Diagnosis of Martin-Bell syndrome based on an analysis of the structural-functional changes in the 5'-untranslated region of the FMR1 gene. Mol. Biol. 33(2), 330-336 (1999).

10. Babenko OV, Zemliakova VV, Saakian SV et al. RB1 and CDKN2A functional defects resulting in retinoblastoma. Mol. Biol. 36(5), 777-783 (2002).

11. Esteller M, Corn PG, Baylin SB, Herman JG. A gene hypermethylation profile of human cancer. Cancer Res. 61(8), 3225-3229 (2001).

12. Tanas AS, Shkarupo VV, Kuznetsova EB et al. Novel tools for unbiased DNA differential methylation screening. Epigenomics 2(2), 325-333 (2010).

13. Kuznetsova EB, Kekeeva TV, Larin SS et al. Novel methylation and expression markers associated with breast cancer. Mol. Biol. 41(4), 624-633 (2007).

14. Győrffy B, Bottai G, Fleischer T et al. Aberrant DNA methylation impacts gene expression and prognosis in breast cancer subtypes. Internat. J. Cancer 138(1), 87-97 (2016).

15. Bock C. Epigenetic biomarker development. Epigenomics 1(1), 99-110 (2009).

16. Lomberk G, Blum Y, Nicolle R et al. Distinct epigenetic landscapes underlie the pathobiology of pancreatic cancer subtypes. Nat. Commun. 9(1), 1978 (2018).

17. Salomon MP, Wang X, Marzese DM et al. The epigenomic landscape of pituitary adenomas reveals specific alterations and differentiates among acromegaly, Cushing's disease and endocrine-inactive subtypes. Clinical Cancer Res. 24(17), 4126-4136 (2018).

18. Pangeni RP, Zhang Z, Alvarez AA et al. Genome-wide methylomic and transcriptomic analyses identify subtype-specific epigenetic signatures commonly dysregulated in glioma stem cells and glioblastoma. Epigenetics 13(4), 432-448 (2018).

19. Shi YX, Wang Y, Li X et al. Genome-wide DNA methylation profiling reveals novel epigenetic signatures in squamous cell lung cancer. BMC Genom. 18(1), 901 (2017).

20. Tanas AS, Kuznetsova EB, Borisova ME et al. Reduced representation bisulfite sequencing design for assessing the methylation of human CpG islands in large samples. Mol. Biol. 49, 618-626 (2015). 
